Methods: The analysis was carried out in plasma and purified leucocytes from 25 subjects, including 12 RA and 13 SLE patients. To evaluate the influence of Bcells depletion on the inflammatory profile of T-cells, purified lymphocytes from 6 RA and 7 SLE patients were treated with RTX $(1 \mu \mathrm{g} / \mathrm{ml})$ for 24 hours. B-cells depletion was assessed by flow cytometry and the changes occurred in the inflammatory profile of T cells were analysed by RT-PCR. The changes promoted in the activity of key intracellular regulators of pro-inflammatory cytokines were analysed by western blot in proteins purified from lymphocytes. In a second set of experiments, supernatants from cultured lymphocytes of 6 RA and 7 SLE patients was added -either, in the presence or in the absence of RTX- to cultured endothelial cells (HUVECs), monocytes, and neutrophils isolated from Healthy Donors (HDs) and incubated for 6 hour. The changes induced in the inflammatory/prothrombotic profile of these cells was analysed by RT-PCR. Finally, serum obtained from 6 RA and 6 SLE patients at baseline and after 3 months of therapy with RTX, was added to HUVECs, monocytes, and neutrophils isolated from HDs and the response was analysed by RT-PCR

Results: In parallel to the significant decline of B-cells, a downregulation of the pro-inflammatory profile of T-lymphocytes from RA and SLE patients was demonstrated, revealed by the significant drop of IL1, IL6, IL17, IFN $\gamma$, and TNF $\alpha$ gene expression levels. A decrease in the phosphorylation status and protein expression levels of STAT-3 and p38 was also found in T-cells treated with RTX. HUVECs, monocytes, and neutrophils incubated with the supernatant of RTXtreated lymphocytes from RA and SLE patients showed a decrease in the expression levels of various pro-thrombotic factors (i.e. TF, IL8, and VEGF) and celladhesion molecules (i.e. V-CAM, I-CAM and e-Selectin). Likewise, HUVECs, monocytes, and neutrophils treated with serum of RA and SLE patients after 3 months of therapy with RTX, showed a reduced expression of genes related to their pro-thrombotic and pro-inflammatory profiles

Conclusions: Depletion of B-cells induced by RTX might promote a beneficial effect in the CV risk-profile of RA and SLE patients through the modulation of the inflammatory and pro-thrombotic shapes of leukocytes and vascular endothelial cells

Acknowledgements: Funded by Junta de Andalucia (CTS-7940) and the Ministry of Health (ISCIII, PI15/01333 and RIER RD16/0012/0015) cofinanced with FEDER funds

Disclosure of Interest: None declared

DOI: 10.1136/annrheumdis-2018-eular.6386

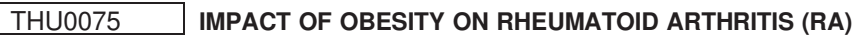 ONSET AND PROGRESSION. IN VIVO AND IN VITRO EFFECTS OF SYNTHETIC DMARDS ON THE RA- ASSOCIATED METABOLIC ALTERATIONS}

1. Arias De La Rosa, M. Ruiz-Ponce, A. Escudero, P. Ruiz-Limón, C. PérezSánchez, M.D.C. Ábalos-Aguilera, Y. Jiménez-Gómez, E. Collantes, C. LópezPedrera, N. Barbarroja. Rheumatology service, IMIBIC/Reina Sofía Hospital/ University of Córdoba, Córdoba, Spain

Objectives: 1) To evaluate the impact of obesity in RA onset and progression, 2) To analyse the in vivo effects of synthetic disease-modifying antirheumatic drugs (sDMARDs) on the obesity and IR in an obese collagen-induced arthritis (CIA) mouse model. 3) To study the in vitro effects of sDMARDs on the lipid and glucose homeostasis in adipose tissue (AT)

Methods: CIA was developed in obese and lean mice. 55 C57BI/6 mice (4-5 weeks) were used. Forty-one mice were fed with high fat diet $(60 \%)$ until reaching $30 \mathrm{~g}$ (obese) (OB). OB-mice were treated with leflunomide (LFN)(10 mg/kg daily), methotrexate (MTX)(3 mg/kg three times/week) or hydroxychloroquine (HCQ) (60 mg/kg daily) for 15 days. After treatment, glucose tolerance test (GTT) was performed. Buffy coat, plasma and adipose tissue (AT) samples were collected. 3 T3-L1 adipocytes were treated with serum from RA patients (10\%) alone or in combination with sDMARDs at day 9 of differentiation. Human subcutaneous AT samples were treated ex vivo with serum from RA patients (10\%) alone or in combination with sDMARDs. Protein and gene expression of molecules involved in inflammation, insulin signalling and lipid accumulation was analysed through RTPCR, western blot and ELISA in all the experiments.

Results: $\mathrm{CIA}-\mathrm{OB}$ mice developed the arthritis earlier and more severe compared with CIA-lean mice. On the hand, arthritis increased the systemic levels of inflammation and HOMA-IR of OB-mice. The induction of arthritis in OB mice increased the inflammatory burden, accompanied by a reduction of genes involved in insulin signalling and lipid accumulation, inducing an aggravation of the insulin resistance (IR) state in AT.

The therapies more effective inhibiting the generation of inflamed digits were $\mathrm{HCQ}$ and MTX. HCQ significantly reduced the body weight, accompanying by a reduction in insulin and glucose plasma levels leading to a decrease of HOMA-IR values. AT of CIA-OB mice treated with MTX and $\mathrm{HCQ}$ had restored levels of genes involved in lipid accumulation, adipogenesis and insulin signalling.
Serum from RA patients elevated the levels of inflammatory markers, reduced the expression of genes related to lipid accumulation, adipogenesis and insulin signalling in 3 T3L1 adipocytes. Although LFN, MTX and HCQ in vitro treatment reduced inflammation, only $\mathrm{MTX}$ and $\mathrm{HCQ}$ regulated insulin sensitivity and lipid accumulation. These results were recapitulated in ex vivo treatments in human AT explants.

Conclusions: 1) Obesity accelerates the development and aggravates the out come of the arthritis in mice. Arthritis exacerbates the inflammatory burden and the metabolic alterations in an obesity context. 2) In vivo, HCQ promotes a beneficial effect on the metabolism of $\mathrm{CIA}-\mathrm{OB}$ mice, improving the insulin sensitivity at systemic and AT levels and reducing body weight. 3) In vitro, HCQ and MTX revert the metabolic alterations induced by RA serum in AT. Thus, HCQ and MTX might be considered as a valuable therapeutic strategy in RA patients to ameliorate the metabolic complications associated.

Acknowledgements: Supported by the Minister of Health (ISCIII, PI17/01316, CP15/0158, RIER RD16/0012/0015) cofinanced with FEDER funds and Roche Pharma, S.A.

Disclosure of Interest: None declared

DOI: 10.1136/annrheumdis-2018-eular.6302

\section{THU0076 ANTIBODIES TO CITRULLINATED PROTEIN ANTIGENS (ACPAS) INDUCE ADIPOSE TISSUE DYSFUNCTION IMPAIRING ADIPOCYTE DIFFERENTIATION, LIPID ACCUMULATION AND PROMOTING MACROPHAGE POLARISATION. IN VITRO EFFECT OF BIOLOGIC DMARDS}

I. Arias De La Rosa, M. Ruiz-Ponce, P. Ruiz-Limón, Y. Jiménez-Gómez, C. PérezSánchez, I. Cecchi, M.D.C. Ábalos-Aguilera, E. Collantes-Estévez, A. Escudero, C. López-Pedrera, N. Barbarroja. Rheumatology service, IMIBIC/Reina Sofía Hospital/University of Córdoba, Córdoba, Spain

Background: Adipose tissue (AT) dysfunction is an important determinant of inflammation- or lipid-induced metabolic complications. Rheumatoid arthritis (RA) is closely associated with metabolic comorbidities such as obesity or insulin resistance (IR). ACPAs are involved in the development of cardiovascular disease associated to this disorder. However, the role of ACPAs on the adipose tissue (AT) function is unravelled.

Objectives: 1) To analyse the direct effects of ACPAs on the AT function: adipocyte differentiation, macrophage polarisation and lipid accumulation, and 2) To evaluate the effects tocilizumab (TCZ) or infliximab (IFX) on the metabolic alterations induced by ACPAs on AT.

Methods: IgGs-NHS (Normal Human Serum) and IgGs-ACPAs were isolated from serum of 20 controls and 20 RA patients. 3 T3-L1 fibroblast were treated with IgG-NHS or IgG-ACPAs alone or in combination with IFX or TCZ during several stages of the differentiation to adipocytes (day 0 and day 9). Lipid accumulation was analysed by oil red 0 (ORO) staining. M0 macrophages from THP-1 cells were treated with IgG-NHS or IgG-ACPAs alone for 12 hour or in combination with IFX and TCZ for another 12 hour. Macrophage polarisation was analysed by flow cytometry. Visceral and subcutaneous AT samples were obtained from 8 obese patients through bariatric surgery. AT samples were treated ex vivo with IgGs-NHS or IgG-ACPAs alone or in combination with biological DMARDs. Protein and gene expression of molecules involved in adipogenesis, inflammation, insulin signalling and lipid accumulation was analysed through RT-PCR, western blot and ELISA in all the experiments.

Results: In vitro treatment of M0 macrophages with IgG-ACPAs induced M1 polarisation state and impaired insulin signalling. 3 T3-L1 fibroblast treated with IgG-ACPAs at day 0 showed an impaired adipocyte differentiation shown by a reduction of genes involved in adipogenesis and lipid accumulation. Levels of accumulated lipids were also significantly reduced. Likewise, genes involved in insulin signalling were reduced. Treatment with IFX and TCZ after differentiation reverted the expression of these genes. At human adipose tissue level, the treatment with lgGs-ACPAs increased the levels of inflammatory markers, accompanied by a downregulation of genes involved in lipid accumulation, adipogenesis and insulin signalling. After treatment with biological DMARDs, inflammatory and metabolic alterations were reverted on human AT explants.

Conclusions: 1) ACPAs impairs AT function, acting in both, macrophages and adipocytes, inducing $\mathrm{M} 1$ macrophage polarisation and impairing adipogenesis and lipid accumulation in adipocytes, favouring an IR state. 2) TCZ and IFX might reverse the metabolic alterations induced in AT by ACPAs. 3) Targeting these autoantibodies would be an excellent therapeutic strategy to restore AT function and reduce the metabolic complications related to $R A$.

Acknowledgements: Funded by the Minister of Health (ISCIII, PI17/01316 and CP15/00158 and RIER RD16/0012/0015) cofinanced with FEDER funds

Disclosure of Interest: None declared

DOI: 10.1136/annrheumdis-2018-eular.6289 